\title{
MicroRNA-33a inhibits lung cancer cell proliferation and invasion by regulating the expression of $\beta$-catenin
}

\author{
CHANGLIANG ZHU $^{1 *}$, YACHAO ZHAO ${ }^{2 *}$, ZHIPEI ZHANG ${ }^{1}$, YUNFENG NI $^{1}$, XIAOFEI LI ${ }^{1}$, and HAN YONG ${ }^{1}$ \\ ${ }^{1}$ Department of Thoracic Surgery, Tangdu Hospital, Fourth Military Medical University, Xi'an, Shaanxi 710038, \\ ${ }^{2}$ Department of Thoracic Surgery, The 309th Hospital of the Chinese PLA, Beijing 100091, P.R. China
}

Received March 9, 2014; Accepted November 19, 2014

DOI: $10.3892 / \mathrm{mmr} .2014 .3134$

\begin{abstract}
MicroRNAs (miRNAs) are short, non-coding RNAs that are aberrantly expressed in tumors. miRNA-33a (miR-33a) is closely associated with cholesterol metabolism and is essential for cellular growth. The aim of the present study was to explore the role of miR-33a and identify its clinical significance in lung cancer cells. miR-33a was observed to be overexpressed in the lung cancer cell lines A549 and NCI-H460. MTT assay results demonstrated that the overexpression of miR-33a significantly inhibited the proliferation of A549 cells, and similar results were obtained from the colony formation assay. This suggests that transfection of miR-33a may suppress the growth of lung cancer cells. Overexpression of miR-33a was also observed to result in marked $G_{1} / S$ phase cell cycle arrest in A549 and NCI-H460 cell lines using fluorescence-activated cell sorting analysis. Western blot analysis revealed that overexpression of miR-33a significantly reduced the expression of $\beta$-catenin in A549 and NCI-H460 cells, suggesting a direct or indirect regulation of $\beta$-catenin by miR-33a in lung cancer cells. In conclusion, the current study may provide strategies for the treatment of lung cancer and clarify the mechanism of its progression.
\end{abstract}

\section{Introduction}

Lung cancer is a disease characterized by uncontrolled cell growth in lung tissue and has the highest incidence and mortality of any type of cancer $(1,2)$. Small cell lung carcinoma (SCLC) and non-small cell lung carcinoma (NSCLC) are the most prevalent types of lung cancer, and $80 \%$ of cases of lung cancer are NSCLC. Following diagnosis with lung cancer, the 5-year

Correspondence to: Mr. Xiaofei Li or Mr. Han Yong, Department of Thoracic Surgery, Tangdu Hospital, Fourth Military Medical University, 1 Xinsi Road, Xi'an, Shaanxi 710038, P.R. China

E-mail: lixiaofeihi@163.com

E-mail: hanhong012@126.com

${ }^{*}$ Contributed equally

Key words: microRNA-33a, lung cancer, $\beta$-catenin, proliferation survival rate for patients is $10 \%(3,4)$, and the incidence and rate of mortality of lung cancer in China is rising. Thus, improvements in clinical diagnosis and management are required. Previous studies have focused on microRNAs (miRNAs) in the pathogenesis and progression of lung cancer (5-8).

miRNAs are a family of small noncoding RNAs that are 20 23 nucleotides in length, and act predominantly at the posttranscriptional level, working as critical regulators of gene expression $(9,10)$. miRNAs are vital in the regulation of cell apoptosis and proliferation, in addition to functioning as either oncogenes or tumor suppressors in the cell cycle. At present, the number of miRNAs encoded by the human genome is $>1,000$, and miRNAs are established to be distributed in various cells and tissues in different proportions $(11,12)$. miR-33a has been identified to be important in the lipid metabolism and the regulation of cholesterol and high-density lipoprotein formation, via the downregulation of ABCA1 and ABCG1 expression (13). SREBP is an important gene that regulates lipid metabolism and the synthesis of fat, while the SREBP/miR-33 locus has been suggested to be involved in cell proliferation and cell cycle progression (14). Cirera-Salinas et al (15) demonstrated that miR-33a was downregulated in lung cancer cells, however, the mechanism of miR-33a in the modulation of lung cancer progression remains to be fully elucidated.

In the present study, miR-33a was overexpressed in the lung cancer cell lines A549 and NCI-H460, and the progression and cell cycles were determined in miR-33a-transfected lung cancer cells. An improved understanding of the progression and mechanisms of lung cancer would be beneficial to patient prognosis.

\section{Materials and methods}

Cells and reagents. The human lung cancer cell lines A549 and NCI-H460 were purchased from the American Type Culture Collection (Manassas, VA, USA) and maintained in our lab in HyClone Dulbecco's modified Eagle's medium (DMEM; GE Healthcare Life Sciences, Logan, UT, USA) supplemented with $10 \%$ fetal bovine serum (FBS; Gibco Life Technologies, Carlsbad, CA, USA). MTT was obtained from Sigma-Aldrich (St. Louis, MO, USA).

Reverse transcription-quantitative polymerase chain reaction $(R T-q P C R)$. The pri-miRNA PCR product was amplified and constructed into a pGenesil-1.1 vector and the sequence was 
A

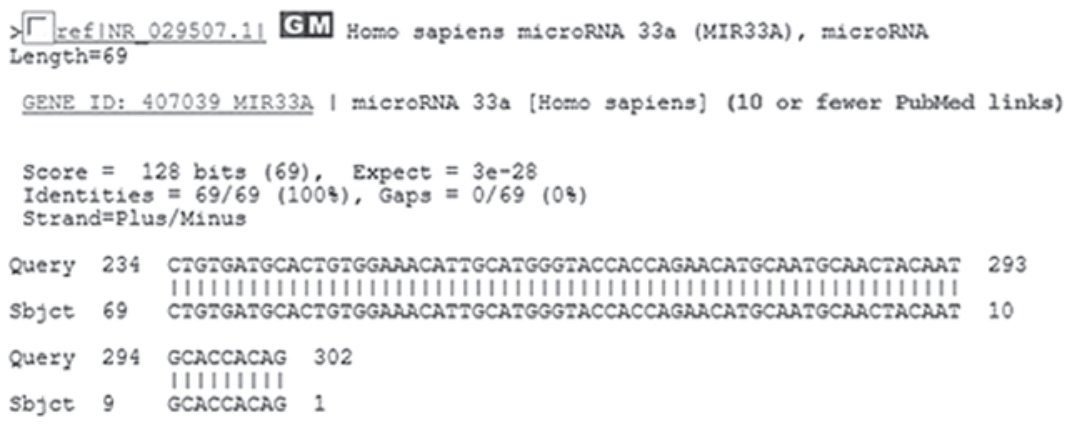

B

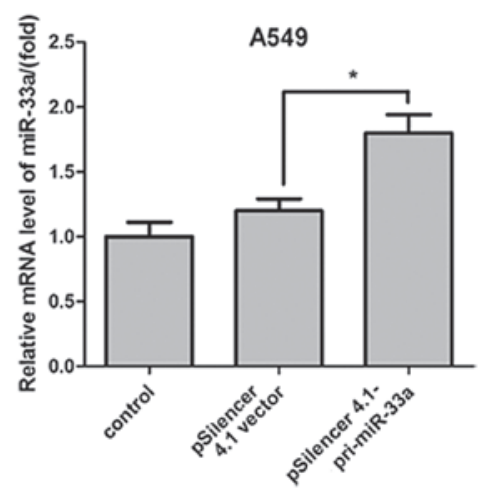

Figure 1. Expression of miR-33a in A549 lung cancer cells. (A) Basic local alignment search tool analysis of recombinant plasmid sequences. (B) Expression levels of miR-33a determined by quantitative polymerase chain reaction.

A

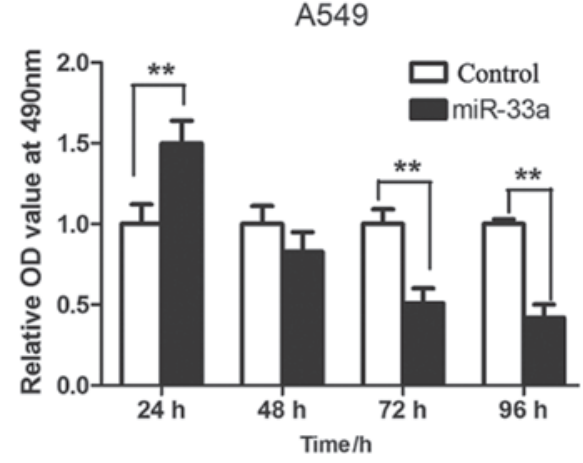

B

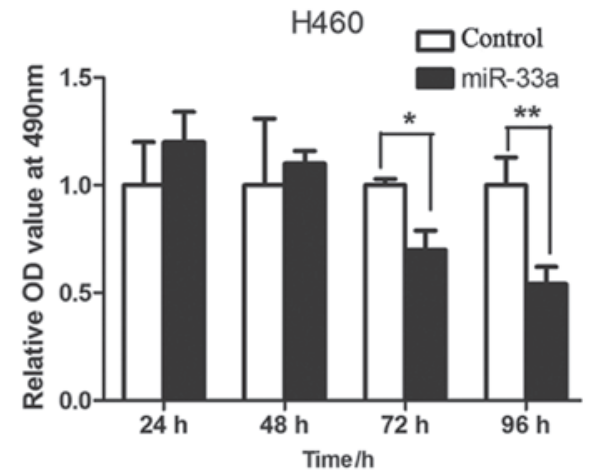

Figure 2. Transfection with miR-33a inhibits the proliferation of A549 and NCI-H460 lung cancer cells. Lung cancer cell lines (A) A549 and (B) NCI-H460 were planted into 48-well plates and cultured for 4 6 h, subsequently the cells were transfected with either the pGenesil-1.1-miR-33a vector or the negative control pGenesil-1.1 vector for $24,48,72$ and $96 \mathrm{~h}$. All data are presented as the mean \pm standard deviation, ${ }^{*} \mathrm{P}<0.05$, ${ }^{* *} \mathrm{P}<0.01$ vs. control. miR-33a, microRNA-33a; OD, optical density.

measured and aligned using the basic local alignment search tool (blast.ncbi.nlm.nih.gov/Blast.cgi; National Center for Biotechnology Information). RT-qPCR was used for detecting mRNA expression levels of miR33a, $\beta$-actin and $\beta$-catenin. Total RNA was extracted and reverse transcribed into cDNA using M-MLV reverse transcriptase and the oligo(deoxythymine) 15 primer, and the resulting cDNA was used as a template for PCR amplification. The primer sequences were as follows: miR-33a, F 5'-GGTTAGATCTTGCTCCAGCGGTTTG-3' and R 5'-GTAAAGCTTGCCCTCCTGTTTCCTG-3'; $\beta$-actin, F 5'-AGAGCTACGAGCTGCCTGAC-3' and R 5'-AGCACT GTGTTGGCGTACAG-3'.

MTT assay. The proliferation of lung cancer cells was measured using an MTT assay as previously described (16-18). Briefly, the A549 and NCI-H460 lung cancer cells were plated into 48-well plates. Following culture for $8 \mathrm{~h}$, the cells were transfected with pGenesil-1.1-miR-33a or the negative control pGenesil-1.1 and cultured for another $6 \mathrm{~h}$. Next, the medium was changed to DMEM with $10 \%$ FBS and the cells were cultured for $24,48,72$ or $96 \mathrm{~h}$. The proliferation of the lung cancer cells was determined by measuring the optical density of the samples at $490 \mathrm{~nm}$.

Clone formation assay. The cells were plated into 6-well plates $\left(5 \times 10^{5}\right.$ cells/well) and transfected with pGenesil-1.1 and pGenesil-1.1-miR-33a cultured for 10 days. The medium was refreshed every 4 days. The surviving colonies ( $\geq 50$ cells/colony) were fixed with methanol. The colonies were stained with $1.25 \%$ crystal violet staining solution (\#C0121; Beyotime Institute of Biotechnology, Jiangsu, China) and counted under a light microscope.

Western blot analysis. The cell extracts were lysed in SDS lysis buffer (\#P0013G; Beyotime Institute of Biotechnology) and separated using SDS-PAGE as previously described (19-21) on $10 \%$ polyacrylamide gel. The SDS buffer consisted of the following reagents: $250 \mathrm{mM}$ Tris- $\mathrm{HCl}(\mathrm{pH} 6.8) ; 10 \%(\mathrm{w} / \mathrm{v})$ SDS; $0.5 \%(\mathrm{w} / \mathrm{v})$ bromophenol blue; $50 \%$ (v/v) glycerin; and $5 \%(\mathrm{w} / \mathrm{v}) \beta$-mercaptoethanol. The antibodies used were as follows: $\beta$-Catenin monoclonal mouse anti-human $\operatorname{IgG}_{1}(12 \mathrm{~F} 7$; sc-59737); $\beta$-actin monoclonal mouse anti-gizzard $\operatorname{IgG}_{1}(\mathrm{C} 4$; sc-47778); goat anti-mouse horseradish peroxidase-conjugated secondary antibody (sc-2005; 1:10,000). All antibodies were obtained from Santa Cruz Biotechnology, Inc. (Dallas, TX, USA).

Flow cytometric analysis. Cell cycle progression was determined using propidium iodide (PI) staining as previously described $(22,23)$. A total of $1 \times 10^{6}$ A549 lung cancer cells were washed twice in cold HyClone phosphate-buffered saline (PBS; GE Healthcare Life Sciences) and fixed in $4 \%$ paraformalde- 

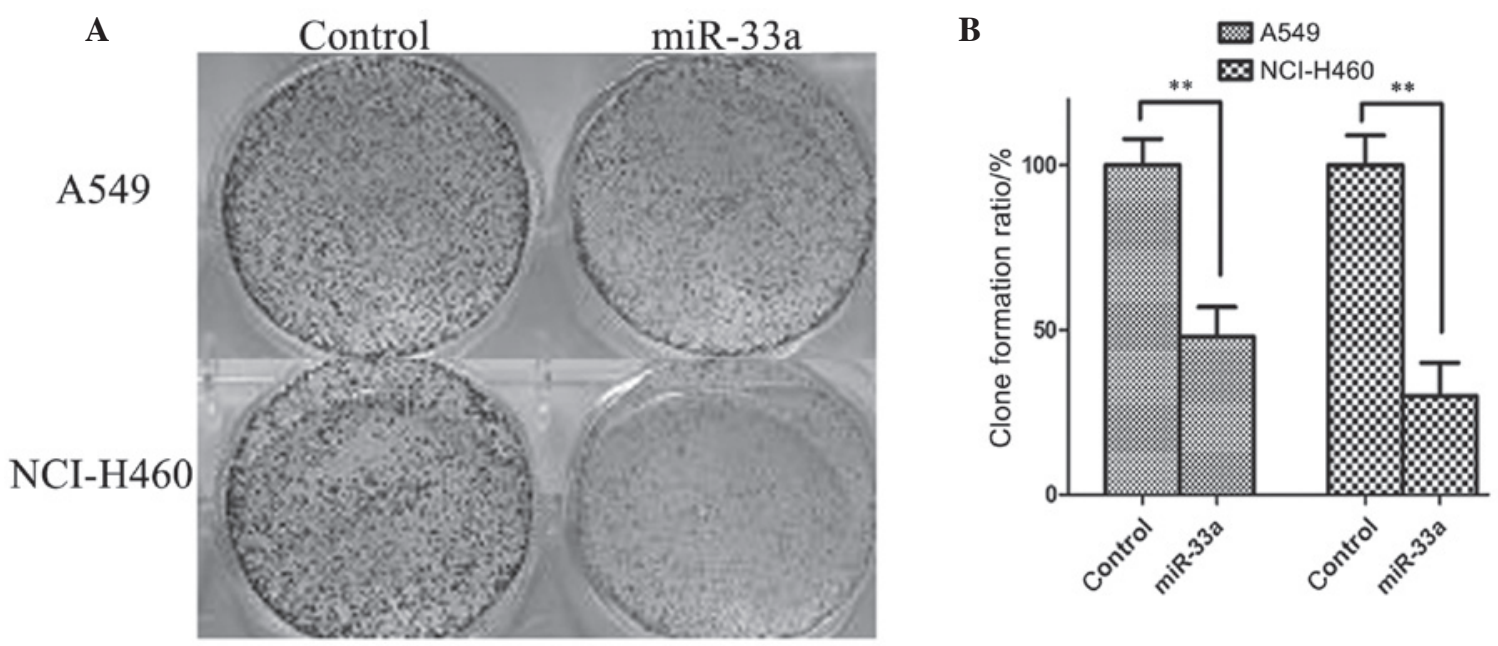

Figure 3. Transfection with miR-33a inhibits the colony formation of lung cancer cell lines. (A) Colonies of A549 and NCI-H460 cells transfected with the pGenesil-1.1 and pGenesil-1.1-miR-33a vectors for 10 days and stained with crystal violet. (B) Histogram of the clone formation ratio. All data are presented as the mean \pm standard deviation, ${ }^{* *} \mathrm{P}<0.01$ vs. control. miR-33a, microRNA-33a.

A

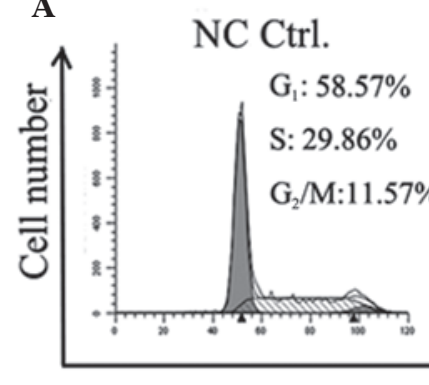

$\operatorname{miR}-33 \mathrm{a}$

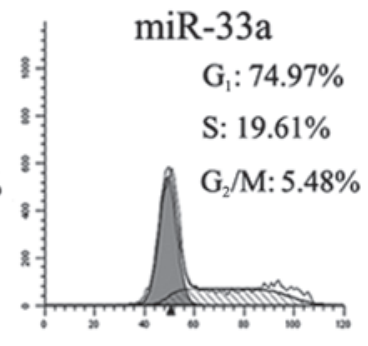

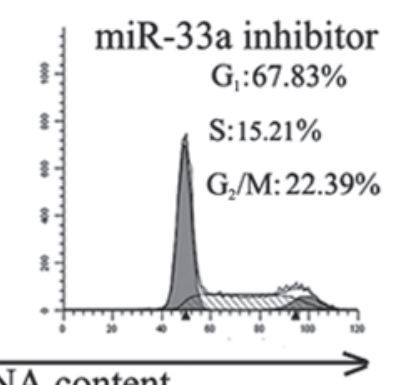

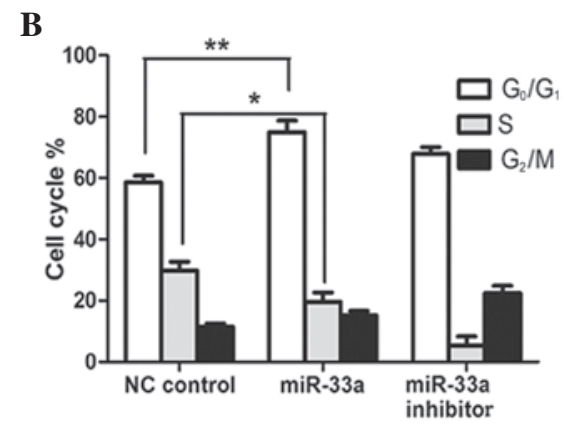

B

Figure 4. miR-33a induces cell cycle arrest at the $G_{1} / S$ phase in lung cancer cells. (A) Cells were stained with propidium iodide and the cell distribution was determined by flow cytometry. (B) Histogram of cell cycle distribution. ${ }^{*} \mathrm{P}<0.05,{ }^{* *} \mathrm{P}<0.01$ vs. NC cells. miR-33a, microRNA-33a; NC, negative control.

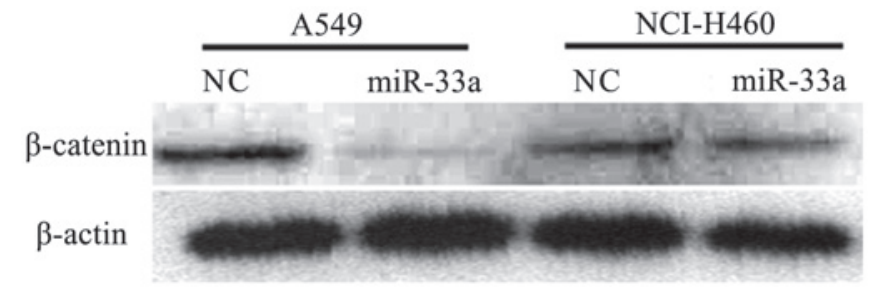

Figure 5. Transfection with miR-33a inhibits the expression of $\beta$-catenin. The lung cancer A549 and NCI-H460 cell lines were transfected with miR-33a and cell lysates were prepared for detecting the expression of $\beta$-catenin by western blot analysis. $\beta$-actin was used as an internal reference. miR-33a, microRNA-33a; NC, negative control.

hyde (Sigma-Aldrich). Following a 30-min resting period, the cells were washed twice again in PBS, PI (Sigma-Aldrich) and RNase A (Sigma-Aldrich) and were added at a final concentration of $100 \mathrm{ng} / \mathrm{ml}$. Subsequent to incubation for $10 \mathrm{~min}$ at room temperature, the cells were detected and analyzed using flow cytometry (Beckman Coulter, Brea, CA, USA).

Statistical analysis. SPSS, version 11.5 (SPSS, Inc., Chicago, IL, USA) was used for statistical analysis and all results are presented as the mean \pm standard error. $\mathrm{P}<0.05$ was considered to indicate a statistically significant difference.

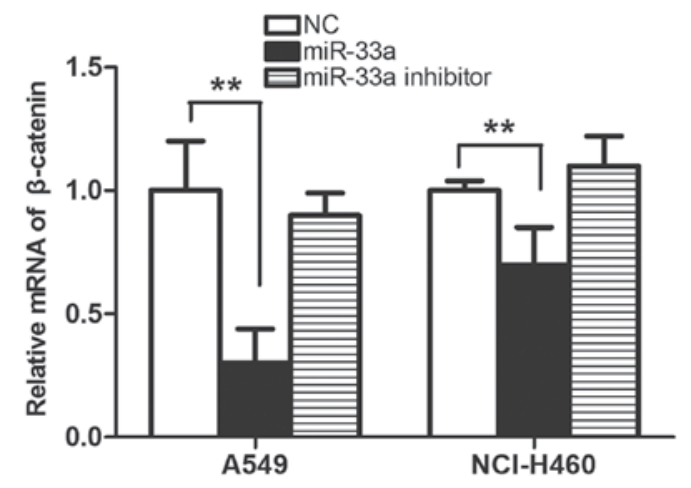

Figure 6. Histogram of relative $\beta$-catenin mRNA levels in A549 and NCI-H460 cells transfected with miR-33a. Expression of $\beta$-catenin was detected by quantitative polymerase chain reaction. Total RNA was prepared and reverse transcribed with M-MLV reverse transcriptase and the oligo(deoxythymine) 15 primer. ${ }^{* *} \mathrm{P}<0.01$ vs. NC. $\beta$-actin was used as an internal reference. miR-33a, microRNA-33a; NC, negative control.

\section{Results}

The expression of miR-33a in A549 lung cancer cells. The pri-miRNA PCR product sequence was measured and aligned using the basic local alignment search tool (Fig. 1A). The expression level of miR-33a was detected in A549 cells using 
qPCR, and the results demonstrated that miR-33a expression was significantly upregulated when A549 cells were transfected with miR-33a for $24 \mathrm{~h}$ (median ratio of 1.8-fold; P<0.05; Fig. 1B) compared with the control vector.

Transfection with miR-33a inhibits the proliferation of the lung cancer cell lines A549 and NCI-H460. In order to detect the effects of miR-33a on proliferation of lung cancer cells, MTT assay was used to measure proliferation levels in the A549 and NCI-H460 cells. The two cell lines were transfected with miR-33a and cultured for 24, 48, 72 and $96 \mathrm{~h}$, and the relative optical density values measured at a wavelength of $490 \mathrm{~nm}$ were determined. The results indicated that the rate of proliferation of miR-33a-transfected A549 cells was significantly lower than the controls at 72 and $96 \mathrm{~h}$ (P<001; Fig. 2A). This was consistent with levels detected in the NCI-H460 cells $(\mathrm{P}<0.05$ and $\mathrm{P}<0.01$, respectively; Fig. 2B).

Clone formation rate is reduced in lung cancer cells transfected with miR-33a. The colony formation assay was used to confirm the inhibitory effect of miR-33a on the lung cancer cell lines A549 and NCI-H460. As illustrated in Fig. 3, the number of colonies formed was significantly reduced in A549 and NCI-H460 cells transfected with miR-33a for $48 \mathrm{~h}$ compared with controls $(\mathrm{P}<0.01)$. Thus, upregulation of miR-33a may inhibit the proliferation of A549 and NCI-H460 lung cancer cells.

miR-33a induces cell cycle arrest at $G_{I} / S$ phase in lung cancer cells. Next, cell distribution was determined by fluorescence-activated cell sorting (FACS) analysis in a different group of cells. As demonstrated in Fig. 4, a significantly greater number of miR-33a-transfected A549 cells were arrested at the $\mathrm{G}_{1} / \mathrm{S}$ phase compared with the negative control group $(\mathrm{P}<0.01)$, with the percentage of $G_{1}$ phase cells increasing from 58.57 to $74.97 \%$.

Transfection with miR-33a reduces the expression of $\beta$-catenin. $\beta$-catenin is established to be involved in cell cycle progression, and its abnormal expression is observed in various tumor cell lines. In order to detect whether the transfection with miR-33a affects the expression of $\beta$-catenin, western blot analysis was used to detect the expression of $\beta$-catenin in lung cancer cell lines transfected with miR-33a. As demonstrated in Fig. 5, in miR-33a-transfected A549 and NCI-H460 cells, the expression of $\beta$-catenin was reduced compared with that in negative control cells. The results from the western blot analysis were consistent with those determined by qPCR, in which the levels of $\beta$-catenin were observed to be reduced in the miR-33a-transfected group compared with the control. ( $\mathrm{P}<0.01$; Fig. 6) The results suggest that transfection with miR-33a may significantly reduce the expression of $\beta$-catenin, which is likely to be involved in the suppression of cell cycle progression.

\section{Discussion}

miRNAs have been identified to serve an important role in the proliferation, apoptosis, metastasis and invasion in lung cancer progression. Abnormal expression of miRNA was first reported in 2004 (24) and mutation, misexpression and altered mature miRNA processing have been suggested to be implicated and involved in tumor progression (25). miRNAs can function as oncogenes or tumor supressor genes in order to regulate tumor progression. For example, miR-494 has been identified to suppress cell proliferation and induce senescence in A549 lung cancer cells (26); miR-21 inhibits growth and promotes apoptosis in the human lung cancer cell line SPC-A1 (27); and miR-378 to significantly modulate human NSCLC progression and angiogenesis. miR-378 was thus suggested as a novel therapeutic target (28).

Metastasis and invasion of tumor cells are factors key to the high mortality rates observed in patients with lung cancer $(29,30)$. In the present study, whether miR-33a inhibits the invasion and migration of lung cancer cells was investigated. The results from the MTT assay demonstrated that transfection of miR-33a effectively inhibited the proliferation and progression of the A549 and NCI-H460 lung cancer cells. The colony formation assay was conducted in order to further confirm the inhibitory effect of miR-33a on the lung cancer cell lines, and the results were consistent with those of the MTT assay.

In the current study, miR-33a was identified to have potential tumor-suppressive activity, as overexpression of miR-33a was demonstrated to inhibit the growth of lung cancer cells. One of the mechanisms of this effect was suggested to be via the induction of $\mathrm{G}_{1} / \mathrm{S}$ phase cell cycle arrest, which was confirmed by the FACS assay. Western blot analysis identified that overexpression of miR-33a in A549 and NCI-H460 cells results in the downregulation of $\beta$-catenin expression, which is established to be involved in cell cycle progression and is abnormally activated in various types of tumor cell $(31,32)$. In conclusion, the results of the current study provide information that may aid in the development of novel therapeutic strategies for lung cancer, in addition to aiding in the elucidation of the antitumor mechanism of miR-33a.

\section{References}

1. Pendharkar D, Ausekar BV and Gupta S: Molecular biology of lung cancer-a review (Review). Indian J Surg Oncol 4: 120-124, 2013.

2. Xue X, Liu Y, Pan L, et al: Diagnosis of multiple primary lung cancer: a systematic review (Review). J Int Med Res 41: 1779-1787, 2013.

3. Blackhall FH, Shepherd FA and Albain KS: Improving survival and reducing toxicity with chemotherapy in advanced non-small cell lung cancer: a realistic goal? (Review). Treat Respir Med 4: 71-84, 2005.

4. Cortés-Funes H: New treatment approaches for lung cancer and impact on survival (Review). Semin Oncol 29 (Suppl 8): 26-29, 2002.

5. Wang XC, Tian LL, Jiang XY, et al: The expression and function of miRNA-451 in non-small cell lung cancer. Cancer Lett 311: 203-209, 2011.

6. Chen Z, Zeng H, Guo Y, et al: miRNA-145 inhibits non-small cell lung cancer cell proliferation by targeting c-Myc. J Exp Clin Cancer Res 29: 151, 2010.

7. Li J, Yang H, Li Y, et al: microRNA-146 up-regulation predicts the prognosis of non-small cell lung cancer by miRNA in situ hybridization. Exp Mol Pathol 96: 195-199, 2014.

8. Salim H, Arvanitis A, de Petris L, et al: miRNA-214 is related to invasiveness of human non-small cell lung cancer and directly regulates alpha protein kinase 2 expression. Genes Chromosomes Cancer 52: 895-911, 2013.

9. Iorio MV and Croce CM: MicroRNA dysregulation in cancer: diagnostics, monitoring and therapeutics. A comprehensive review (Review). EMBO Mol Med 4: 143-159, 2012. 
10. $\mathrm{Li} \mathrm{YJ}, \mathrm{Li} \mathrm{ZY}$ and $\mathrm{Xu} \mathrm{KL}$ : MicroRNA as potential target for genetherapy of multiple myeloma-review (Review). Zhongguo Shi Yan Xue Ye Xue Za Zhi 21: 1318-1325, 2013.

11. Piva R, Spandidos DA and Gambari R: From microRNA functions to microRNA therapeutics: novel targets and novel drugs in breast cancer research and treatment (Review). Int J Oncol 43: 985-994, 2013.

12. An J, Zhu X, Wang H and Jin X: A dynamic interplay between alternative polyadenylation and microRNA regulation: implications for cancer (Review). Int J Oncol 43: 995-1001, 2013.

13. Wijesekara N, Zhang LH, Kang MH, et al: miR-33a modulates ABCA1 expression, cholesterol accumulation, and insulin secretion in pancreatic islets. Diabetes 61: 653-658, 2012.

14. Gharipour M and Sadeghi M: Pivotal role of microRNA-33 in metabolic syndrome: A systematic review (Review). ARYA Atheroscler 9: 372-376, 2013.

15. Cirera-Salinas D, Pauta M, Allen RM, et al: Mir-33 regulates cell proliferation and cell cycle progression. Cell Cycle 11: 922-933, 2012.

16. Bernas T and Dobrucki J: Mitochondrial and nonmitochondrial reduction of MTT: interaction of MTT with TMRE, JC-1, and NAO mitochondrial fluorescent probes. Cytometry 47: 236-242, 2002.

17. Sylvester PW: Optimization of the tetrazolium dye (MTT) colorimetric assay for cellular growth and viability. Methods Mo Biol 716: 157-168, 2011

18. Campling BG, Pym J, Baker HM, Cole SP and Lam YM: Chemosensitivity testing of small cell lung cancer using the MTT assay. Br J Cancer 63: 75-83, 1991.

19. Nishitani H, Sugimoto N, Roukos V, et al: Two E3 ubiquitin ligases, SCF-Skp2 and DDB1-Cul4, target human Cdt1 for proteolysis. EMBO J 25: 1126-1136, 2006.

20. Peng L, Xu Z, Zhou Y, Yang T, Liang ZQ and Zhang M: Effect of rosiglitazone on cells cycle, apoptosis and expression of Skp2 and p27Kip1 in hepatocellular carcinoma cell line. Zhonghua Gan Zang Bing Za Zhi 18: 148-149, 2010 (In Chinese).

21. Schulman BA, Carrano AC, Jeffrey PD, et al: Insights into SCF ubiquitin ligases from the structure of the Skp1-Skp2 complex. Nature 408: 381-386, 2000
22. Krishan A: Rapid flow cytofluorometric analysis of mammalian cell cycle by propidium iodide staining. J Cell Biol 66: 188-193, 1975.

23. Buchegger F, Dupertuis YM and Perillo-Adamer F: A pitfall of propidium iodide staining in fluorescence-activated cell sorting cell cycle analysis? Cancer Res 67: 5576-5577, 2007.

24. Takamizawa J, Konishi H, Yanagisawa K, et al: Reduced expression of the let-7 microRNAs in human lung cancers in association with shortened postoperative survival. Cancer Res 64: 3753-3756, 2004.

25. Hu Z, Chen J, Tian T, et al: Genetic variants of miRNA sequences and non-small cell lung cancer survival. J Clin Invest 118: 2600-2608, 2008.

26. Ohdaira H, Sekiguchi M, Miyata K and Yoshida K: MicroRNA-494 suppresses cell proliferation and induces senescence in A549 lung cancer cells. Cell Prolif 45: 32-38, 2012.

27. Zhao G, Guo W, Zhao X, Wang Y and Hou Y: Glossy ganoderma spore oil promotes apoptosis of human lung adenocarcinoma SPC-A1 through downregulation of miR-21. Zhongguo Zhong Yao Za Zhi 36: 1231-1234, 2011 (In Chinese).

28. Skrzypek K, Tertil M, Golda S, et al: Interplay between heme oxygenase-1 and miR-378 affects non-small cell lung carcinoma growth, vascularization, and metastasis. Antioxid Redox Signal 19: 644-660, 2013

29. Nonaka M, Kataoka D, Yamamoto S, et al: Outcome following surgery for primary lung cancer with interlobar pleural invasion. Surg Today 35: 22-27, 2005.

30. Hsu YL, Wu CY, Hung JY, Lin YS, Huang MS and Kuo PL: Galectin-1 promotes lung cancer tumor metastasis by potentiating integrin $\alpha 6 \beta 4$ and Notch1/Jagged 2 signaling pathway. Carcinogenesis 34: 1370-1381, 2013.

31. Ding J, Ge D, Guo W and Lu C: Diazoxide-mediated growth inhibition in human lung cancer cells via downregulation of beta-catenin-mediated cyclin D1 transcription. Lung 187: 61-67, 2009.

32. Xiong F, Jiang M, Huang Z, et al: A novel herbal formula induces cell cycle arrest and apoptosis in association with suppressing the PI3K/AKT pathway in human lung cancer A549 cells. Integr Cancer Ther 13: 152-160, 2014. 